\title{
Energy Efficient Mobility in Wireless Sensor Network
}

\author{
Vasaki Ponnusamy, Azween Abdullah \\ Computer \& Information Sciences Department \\ Universiti Teknologi PETRONAS, Malaysia
}

\begin{abstract}
Wireless sensors are used in many areas such as environmental and habitat monitoring, indoor climate control, medical diagnostics, intelligent alarms, surveillance and many more. Since sensor nodes are placed in remote areas and require less attention and supervision, a significant number of these nodes require battery power to survive long periods of time. Recharging or replacing the battery is often difficult since most of these units are placed in areas which are difficult to access. Energyconstrained sensors deplete their energy quickly due to routing sensor data to the base station and also sensing the event. Energy depletion at these nodes causes routing hole and reduces network lifetime. This paper introduces the method of exploiting mobile nodes in the sensor network to increase network lifetime and node lifetime. This mobile nodes, known as mobile agents act as routing agents to increase network lifetime as well as move in position searching for energy, recharge and deliver the harvested energy to static energy depleted nodes. The mobile agent proposed in this paper travels between the static sensor nodes and base station in order to collect sensed data from the sensor nodes and relay it to the base station. Moreover the mobile agent also performs energy harvesting to increase node lifetime. The proposed architecture is inspired by plant biology (botany) based mechanism of the symbiosis interaction between plant root system and microbes in the rhizosphere (soil)
\end{abstract}

\section{Introduction}

As sensor nodes are highly resource constrained, energy efficient communication becomes one of the main issues to be dealt with. Energy-constrained sensors deplete their energy quickly due to routing sensor data to the base station whilst sensing the event. Most of the proposed solution [1][2] looks into using static sensor nodes and multi-hop routing to convey sensed data. The use of multi-hop communication is a primitive WSN operation that is extremely fault-prone as well as energy-intensive.
A self-healing architecture is needed in order to allow sensor networks to heal from failures and resume its service. Self-healing network must take into consideration the severe resource limitation (energy) of sensor networks. [3] Looks into a new concept of introducing mobile agent. It takes the processing load away from low powered sensors and uses hierarchical architecture. It is more energy efficient but does not look into having mobile agent as routing agent and reducing multi-hop operation. SASHA [1] is a self-healing hybrid sensor network using natural immune system concepts that uses automatic fault recognition and response. This protocol only looks at faulty sensor readings and does not look into approach of reducing energy conservation by means of self-healing.

One of the potential problems with current routing protocols is that, it looks for lowest energy route and uses that route for every communication. And this causes energy depletion along the path as this is not good for network lifetime [3]. Moreover existing mechanisms rarely discuss on the crosscutting matters such as energy consumption, errorchecking etc [4]. Therefore an energy efficient routing protocol is needed for the network to selfheal from energy depletion and routing failure. Selfhealing network must take into consideration the severe resource limitation (energy) of sensor networks [1].

\section{Methodology}

The many reasons for failure in sensor network such as energy exhaustion, malicious destruction and malfunction [5] causes the destruction of coverage and connectivity. The failure also depends on the nature of application, its density, nodes closer to the base station and environmental conditions. Some nodes closer to the base station die faster as it forwards greater number of data to the base station. All this effects on coverage and connectivity creates a coverage hole along the network. This is also known as routing hole, whereby sensor nodes fail and causes a gap in the network where data cannot be forwarded.

Therefore we propose a self-healing mechanism whereby the node failure due to energy depletion is 
handled by proposing the use of mobile agent (MA). As stated by [6], one of the major advantages of mobile wireless sensor network (WSN) over static WSN is its efficient energy utilization. The mobile WSN proposed in this paper chooses an optimal mobility pattern so that nodes closer to the sink are not utilized the most and this will prevent routing hole. The entire data aggregation and forwarding of data to a base station is taken care by mobile nodes itself. [5] Proposes a new mechanism whereby sensor nodes utilize the remaining energy by relocating to combat the coverage holes. The nodes are mobile by relocating to a location with coverage holes, hence resulting in WSN healing. This approach relives the burden of sensor nodes from using multihop routing to relay data which would eventually cause sensor nodes failure near the base station as discussed. Moreover the mobile nodes do not need to visit every single node but to only obtain data from the cluster head node. better targeting and quality of communication is achieved.

As seen in figure 1, the proposed architecture comprises of three layers. Layer 1 consists of all static sensor nodes deployed, mobile agent sits at layers 2 and layer 3 consists of the base station. Mobile agents at layer 2 can be a laptop, mobile phone, mobile sensor on airplane, robot etc depending on the nature of the setup. The proposed architecture is inspired by plant biology-based (botany) communication. The plant biology communication in this context mainly looks at the mass communication that takes place at plant rhizosphere (sensor area), an information superhighway underground.

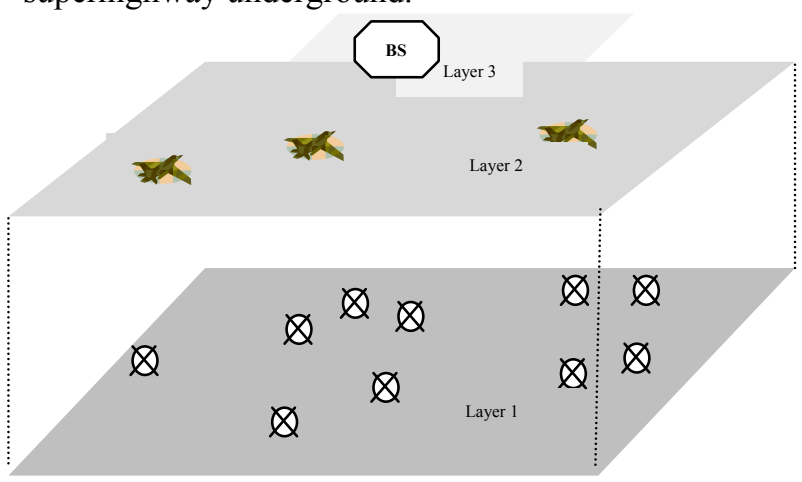

Figure 1. Mobile agent based architecture in WSN

The plant (sensor) using its root system communicates with other plant root systems in the rhizospehere. The entire three-tier architecture is inspired by the nature of how plant root system discover their neighbors, form a colony, create a defense mechanism against other plants or intruder which is known as allelopathy and symbiosis relationship with other micro-organism such as microbe (mobile agent), fungi and bacteria.

The proposed architecture should look into consideration the following aspects: a middleware node is responsible for communication between sensor nodes and base station, short range communication with base station to reduce the distance ie. shortest path, avoid routing hole as much as possible, maintain fault-tolerance and hierarchical in nature. Sensor networks discover their neighbor through a chemical messenger to create a chemotactic relationship. Upon discovery, sensor nodes create a cluster and elect a cluster head for communication purposes. As mentioned above, the middleware proposed in this paper consists of mobile agents (microbe). The mobile agent creates a symbiosis relationship with sensor nodes to receive sensed data. Figure 1 shows how a mobile agent(middleware) helps in this context to convey data from the cluster head to the base station. These proposed MA helps in terms of short range communication thus avoids the routing hole issues. This is because the use of MA instead of cluster head or sensor nodes for forwarding sensed data makes sure that sensor node do not deplete energy quickly. MAs are more powerful units which can have higher battery lifetime.

Mechanisms that save energy should be identified in order to extend the life of the network. Energy harvesting is another issue that has to be taken into consideration in order to extend the life of the network [7]. There have been many researches going on in environmental power scavenging techniques [8] and [9] also work on energy replenishment with the use of mobile robots in sensor network. [10] Proposes a method by having mobile robots as energy producers. These nodes recharge themselves by moving to locations with abundant energy supply. Once they have enough energy, they migrate to areas in the network for delivering energy to the static sensor nodes. In this method a small percentage of network nodes are mobile by allowing them to move to search for energy, recharge, and deliver energy to immobile, energy-depleted nodes. This approach is known as energy harvesting or energy scavenging.

\section{Nature Inspired Framework}

The biological system can be mapped well to communication system and more applicably to the focus of this paper on self-healing characteristics. The mechanisms in this context include balance on the internal equilibrium (homeostasis) and on the self-organization mechanism to support environmental changes. During the lifespan of the WSN communication system, various changes occur such as energy depletion, shortening lifespan, external threat or route diversion. Based on these 
changes, the system should be able to self-learn, selforganize and self-heal from such events. These selfmanagement activities are similar to living organisms such as human, animals and plants whereby they learn and reform (self-heal) [11].

The plant based biologically inspired mechanism consists of three layers as is proposed in figure 1 for sensor network. i) layer 1 consists of plants with its root mechanism and coordination with the rhizoshere, ii) layer2 consists of the communication of the plant with the microbe organisms in the rhizosphere and iii) layer 3 consists of communication of the microbe organisms with the base station. This architecture is well mapped to the sensor network in which the plant system is associated with sensor nodes and microbe organism is associated with the mobile agent.

As can be seen in figure 2, the operation of plant biology as in this context mainly happens at the plant rhizosphere which is the soil mechanism. The plant leaf (sensor node) is exposed to sensing environment to continuously seek for the event. The sensing environment is associated with sun light and its natural resources. Plant uses light from the sun to do something beyond what science can think of: it takes the molecules of carbon dioxide and water and compounds them together to make carbohydrates. Plant leaves get sunlight to produce carbon ion (sensed data) through photosynthesis process. The carbon ions from the leaf then are transported to other parts of the plant and mainly to the root system.

\subsection{Defense Mechanism}

Plants also have the capability of continuously communicating with the surrounding root systems of neighboring plants and can respond quickly to any invading roots of other species plants through chemical messengers. This allelopathic communication is triggered through pythotoxins secreted by plant roots in order to maintain a terrestrial plant community. This mechanism can be applied to sensor network where sensor nodes communicate with others and avoid the intrusion of any threats or possible attacks. This is one of the

\subsection{Neighbor Discovery}

The root system in the rhizhosphere detects each other through a neighbor discovery process as similar to sensor network in which sensor nodes detects each other through a neighbor discovery process. Moreover, plants have the capability of detecting plants of their same colony and form a cluster of community. They can send messages to one another of any possibilities of surrounding attacks, or threats by other animals from other colony. The plant root system is also very sensitive to overcrowding by the same or other species. The same concept is applicable to sensor network where sensor nodes are always sensitive to any possible threats or changes in the environment that is being sensed. Sensor networks can be programmed to form a cluster with homogenous nodes and avoid any possibilities of overcrowding of sensor within the same proximity of area.

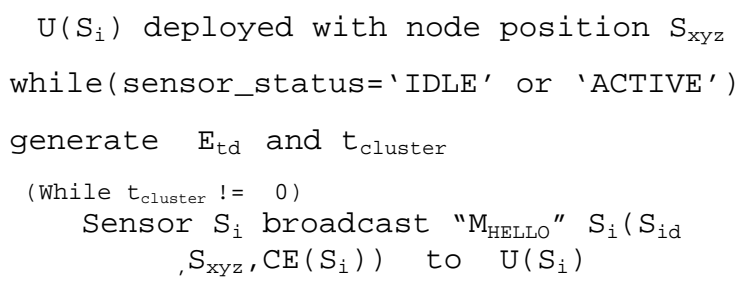

The chemical secretion into the rhizosphere creates a chemotactic relationship between organisms in the soil area which can be a positive or negative reaction. The positive reaction is called symbiotic relation such as the association of microbe

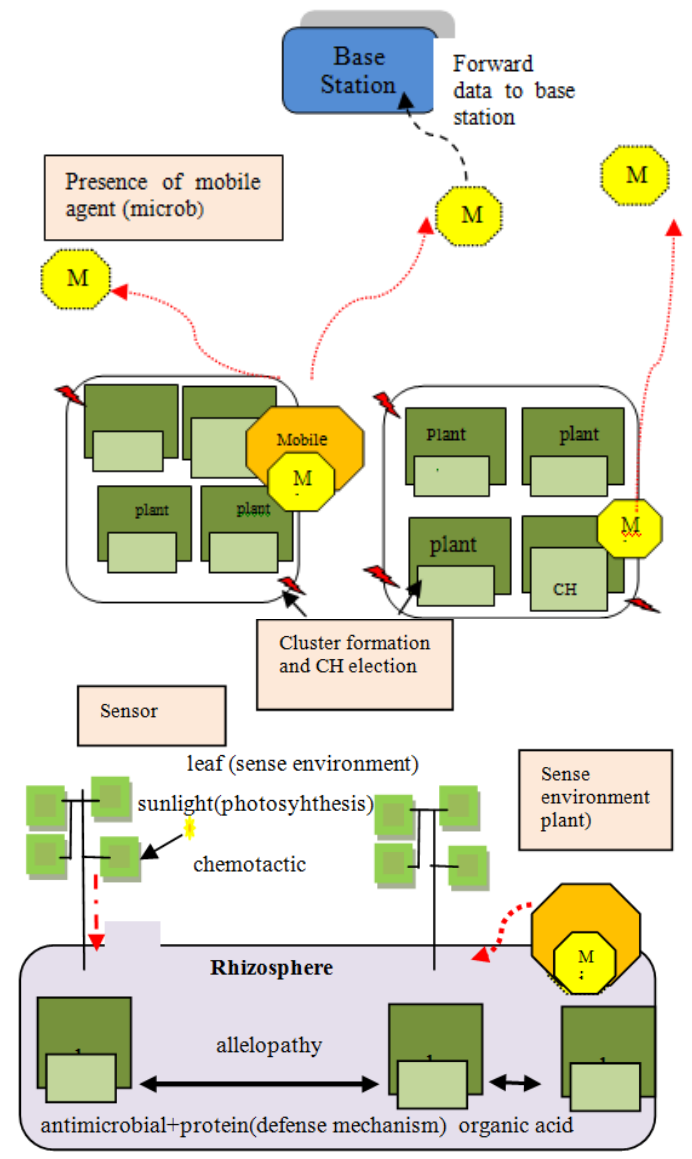

Figure 2. Plant Biology Inspired Framework for WSN 
or fungi and nitrogen-fixing bacteria. These reactions are through organic acid and amino acids (communication messages) secreted by plant roots. The negative reaction includes interactions with parasitic plants and insects [12]. The positive communication is associated with communication among sensor nodes and mobile agents visiting the sensor area to collect sensed data. The sensor nodes and mobile agents communicate through messages (amino acid) exchanged between them.

\section{Symbiosis Communication}

The next layer of communication is the symbiosis relationship between plants (sensor nodes) and microbe (mobile agent) living in the soil rhizosphere. This symbiotic relationship is a two way dependency where plants depend on microbe to process water in the soil and recycle them back into foodstuffs for plant growth and vice versa whereby microbe depends on the plant by root exudates as a main component of food. The interchange of compounds between these two organisms provides a mechanism of warning signals [12]. These associations can be seen in a communication between sensor nodes and mobile agents where a sensor node typically sends sensed data to the mobile agent and the mobile agent depends on the sensor node to collect and forward data.

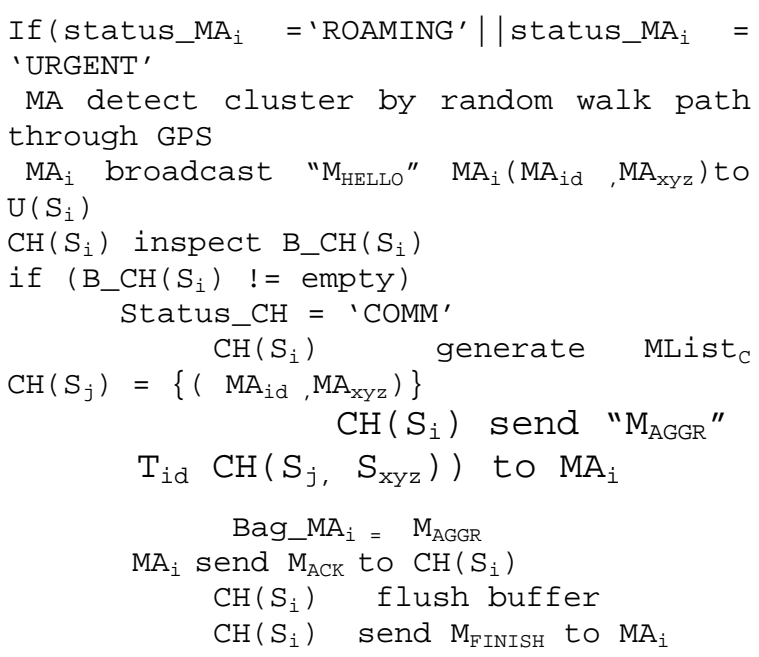

*Where $M_{\text {HELLO }}$ and $M_{\text {FINISH }}=$ organic acid (for neighbor discovery)

This idea also inspires the concept of energy harvesting and scavenging whereby mobile sensors (microbe) can recharge energy at sensor nodes (plant). The energy harvested by mobile agent can be transferred to the sensor nodes. Mobile agent helps sensor nodes to preserve the battery resource by acting as a middleware to forward sensor data to the base station. Sensor nodes self-heal from energy depletion and this increase the network lifetime.

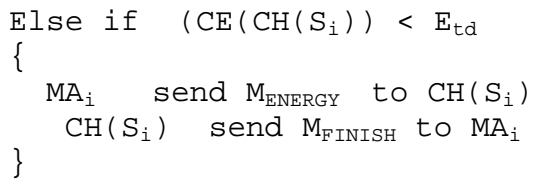

Migration

Microbes are always mobile as in when there is scarce supply of food or nutrient from current symbiotic plant, it moves to other neighboring plants to acquire it. This natural selection mechanism happens to mobile agents whereby it moves from cluster to cluster in order to collect sensed data. Once enough information is received, mobile agent then would move to the base station in order to forward to the base station.

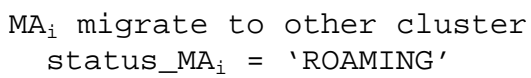

\section{Protocol Overview}

The following section discusses the operation of the protocol in detail by using the ideas of mobile agent and the three-tier architecture.

\subsection{Layer1: sensor node to sensor node communication: cluster formation}

This is the first phase where sensor nodes perform a neighborhood discovery in order to detect the presence of one another. The neighborhood discovery process is important so that sensor nodes could take part in the formation of cluster. And this process helps in the election of cluster head $(\mathrm{CH})$ for a particular cluster. Also nodes discover their neighbor for future reference, if there incurs any possible threats and to prepare for a defense mechanism amongst sensor nodes. Sensors within inter and intra cluster can communicate with each other to form any future security and defense mechanisms. The following is how the protocol and the algorithm are devised:

i. $S \rightarrow S:$ Upon start, sensor nodes starts a timer and send a hello message to each other notifying its presence.(message type=000). Hello message is sent to all sensors within a hop distance of $h$ (cluster), the value $h$ is determined based on signal strength. All sensor nodes start with the same amount of energy. The hello message consists of sensor node identification, GPS location of sensor node and remaining energy level.

- Upon receiving hello message, all sensor nodes create a member list of nodes attached within hop distance $h$. All the fileds in the hello message is copied into the membership list.

The membership list appears as such: 
$\left\{\left(1, X_{1} Y_{1}, E_{1}\right),\left(2, X_{2} Y_{2}, E_{2}\right),\left(3, X_{3} Y_{3}, E_{3}\right)\right\} \quad-1$ refers to node identification, $X Y$ refers to GPS position and $E$ refers to the remaining energy level.

ii. S: Sensor nodes inspect its member table to decide which node has the highest amount of energy compared to the threshold value to be the cluster head. If other nodes within the member table have higher energy than threshold, then do nothing. Else if it has the highest energy level, then elect self to be the cluster head $(\mathrm{CH})$.

iii. $\mathrm{CH} \rightarrow \mathrm{S}: \mathrm{CH}$ broadcasts Sink-up message (message type $=001)$ to other sensors within hop distance $h$ to announce its decision to be the cluster head.

iv. $S \rightarrow C H$ : Sensor nodes decide which cluster it wishes to join and send back a join message(message type $=010)$ to $\mathrm{CH}$.

v. $\mathrm{CH}$ : $\mathrm{CH}$ updates its member list of all nodes belongs to cluster and creates a schedule for all sensor nodes above the threshold value to send data based on the schedule created. All other sensor nodes below the threshold value goes into sleep mode. The structure of $\mathrm{CH}$ member list is:

$\left\{C H=5,\left(1, X_{1} Y_{1}, E_{1}\right),\left(2, X_{2} Y_{2}, E_{2}\right),\left(3, X_{3} Y_{3}, E_{3}\right)\right\}$ whereby the $C_{-} H_{I}$ is 5 and the remaining list is the same as sensor member list created earlier.

vi. $S \rightarrow C H:$ Sensor node senses event and sends data to the CH based on the schedule created.

vii. $\mathrm{CH}$ : $\mathrm{CH}$ creates a buffer to store all the messages being received from sensor nodes. Data aggregation is performed at this point whereby $\mathrm{CH}$ filters redundant data and also compares data from all sensors to have a complete collection of data.

When sensor timer reaches a determined value, step $i$. starts all over again by all sensor nodes, within hop distance $h$ (including sensor in sleep mode) sending a hello packet to each other.

Note: For the sink message and join message, the GPS position and remaining energy is set to nil since sensors have already obtained these information from the hello message and already in the member list. This will save overhead of the message

\subsection{Layer 2: sensor node to mobile agent communication}

This is the layer 2 communication whereby mobile agent communicates with the sensor node. Mobile agents are always in the moving position and its location information is continuously tracked using GPS. Mobile agents have the capability of detecting GPS position of sensor nodes within the neighborhood and the reverse is true whereby when a mobile agent comes into contact with the cluster, the cluster head is able to detect the presence of mobile agent. For the initial round, the order of cluster visits by MA takes place in random fashion after which
MA learns to visit only clusters within nearer proximity. This is possible by all MA maintaining a path visit list.

i. $\mathrm{MA} \rightarrow \mathrm{CH}$ : When mobile agent detects the presence of sensor nodes (based on GPS location), it sends a hello message consisting of GPS location and mobile agent ID to the sensor nodes.

ii. $C H \rightarrow M A$ : Upon receiving this message, only the current cluster head will respond to mobile agent. Before communicating, cluster head inspects its data bag to decide whether there is any data to be sent. If the bag is empty, then $\mathrm{CH}$ will not respond to $M A$. Otherwise $\mathrm{CH}$ responds to $M A$ by sending message that consists of $\mathrm{CH} I D$, cluster member list and the complete aggregated data from the data bag. CH keeps MA node identification in member list for future communication.

iii. MA : MA stores membership list as path visited $\left\{C H=5,\left(1, X_{1} Y_{1}, E_{1}\right),\left(2, X_{2} Y_{2}, E_{2}\right),\left(3, X_{3} Y_{3} E_{3}\right)\right\}$

iv. $\mathrm{CH}$ : After sending the data, $\mathrm{CH}$ will flush the buffer (data bag) to empty the old data and create space for new data from sensors.

v. Upon inspecting the energy level of nodes in the membership list, MA decides nodes that need to be recharged. In this case MA moves to the closer proximity of this node and perform recharging.

vi. MA : MA waits for a random period of time and if no more data being received, MA then decides to move to other clusters which enables MA to piggyback data from. Before leaving the cluster, MA informs $\mathrm{CH}$ about its migration. This is to ensure that $\mathrm{CH}$ does not send anymore data to this MA.

vii. $\mathrm{CH}$ : Upon receiving the migration information, $\mathrm{CH}$ removes $M A$ identity from its member list and looks for other MA that comes within the range of communication.

viii. MA : MA moves to other clusters to piggyback more data and the same process from $i$. to vii. is applied in this context.

$M A$ : once the data bag of MA is full, MA decides to deliver the message to base station, and moves towards the base station

\section{Energy Analysis of various mechanism}

Researchers are looking into possibility of shortening transmission distance using various methods such as i. shorter routing path using multihop routing ii. using cluster head [13], iii. using mobile relay or agent [14], iv. using message ferrying approach and v. mobile sink to reduce the distance. The mathematical analysis presented in this paper, looks at 5 different approaches looking into total energy consumption pattern for energy consumption using i. multihop routing, ii. cluster 
head, iii, direct transmission, iv. mobile agent with cluster head, and v. mobile agent with direct transmission(refer to figure 3). We are analyzing the energy patterns looking into the energy consumed at nodes located nearer to the base station. These nodes assumed to be consuming more energy since it has to forward data that comes from other nodes from other parts of the network as well (for multihop communication). Since the base station is assumed to be located at the centre of the network, more traffic is concentrated there. The analysis presented will prove that mobile agent based routing mechanism could be a potential solution for energy efficient operation.

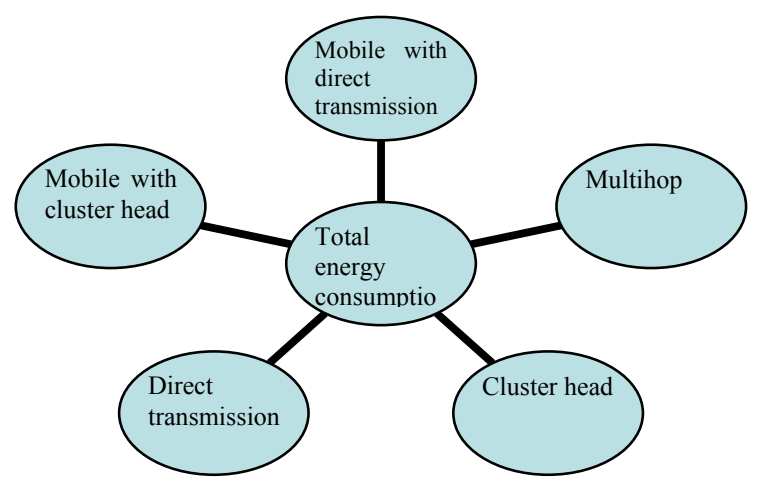

Figure 3. Category of energy consumption analysis

This energy model is extracted from [17,18] and LEACH [13] also considers similar model for its first order radio model. The load in the network is calculated based on the analysis performed by [19 ].

Assume $_{\hat{C}}=$ Energy $_{\text {cpu }} \partial=$ Energy $_{\text {elec }} \quad \mu=$ Energy $_{\text {amp }}$

Energy consumption using multihop

$$
\begin{aligned}
& \left.=\frac{\left(\mathbf{L}^{2}-1\right.}{\pi r^{2}}\right) \partial X a+\frac{\left(L^{2}\right) \partial}{\pi r^{2}}+\mu X d^{2} X a+\hat{C} X a \\
& =a \frac{\left(L^{2}\right)}{\pi r^{2}} \partial-\partial \frac{\left(L^{2}\right)}{\pi r^{2}} \partial+\frac{\left(L^{2}\right) \mu}{\pi r^{2}} X d^{2}+\hat{C} \\
& =a \frac{\left(2 L^{2}\right)}{\pi r^{2}} \partial+\frac{\left(L^{2}\right)}{\pi r^{2}} \mu X d^{2}+\hat{C}
\end{aligned}
$$

Energy consumption using cluster head

$$
\begin{aligned}
& =\mathbf{a}\left(2 \pi L r^{2}\right) \partial+\left(\pi L r^{2}\right) \mu X d^{2}+\hat{C} \\
& \approx a\left(\mu X d^{2}+2 \partial+{ }_{C}\right)
\end{aligned}
$$

Energy consumption using direct transmission

$$
\begin{aligned}
& \text { Total Energy Consumption }=\text { Energy }_{\text {trans }}+\text { Energy }_{\text {sense }} \\
& =\mu \boldsymbol{X} \boldsymbol{d}^{2} \boldsymbol{X} \boldsymbol{a}+\partial \boldsymbol{X} \boldsymbol{a}+{ }_{\hat{C}} \boldsymbol{X} \boldsymbol{a} \\
& =\boldsymbol{a}\left(\mu \boldsymbol{X} \boldsymbol{d}^{2}+\partial+\hat{O}\right)
\end{aligned}
$$

Energy consumption using mobile with cluster head

$$
\begin{aligned}
& =\frac{\left(2 \pi L r^{2}\right)}{\mathbf{N}^{2}} \partial X a+\frac{\left(\pi L r^{2}\right)}{\mathbf{N}^{2}} \mu X H^{2} X a \\
& \left.=\frac{a\left(2 \pi L r^{2}\right.}{\mathbf{N}^{2}} \partial+\frac{\left(\pi L r^{2}\right)}{\mathbf{N}^{2}} \mu_{p} X H^{2}\right)
\end{aligned}
$$

where $\mathbf{H}<\mathbf{r}$

Energy consumption using mobile with direct transmission

$$
\begin{aligned}
& \text { Total Energy Consumption }=\text { Energy }_{\text {trans }}+\text { Energy }_{\text {sense }} \\
& =\mu X H^{2} X a+Ә X a+{ }_{C} X a \text { where } H<r \\
& =\frac{a L^{2}}{\pi r^{2}}\left(\mu X H^{2}+\oslash+{ }_{C}\right)
\end{aligned}
$$

The following section discusses some of the outcome or results obtained from simulation performed by other researchers by comparing with the mathematical analysis presented here. The results obtained are from various approaches such as clustering concept, use of mobile agent, biological inspired concepts and direct transmission. These results will help in future simulation of the protocol mentioned in section 4 . The results obtained by using mobile agent to cover routing hole and energy harvesting should produce better results compared to the results shown by other approaches.

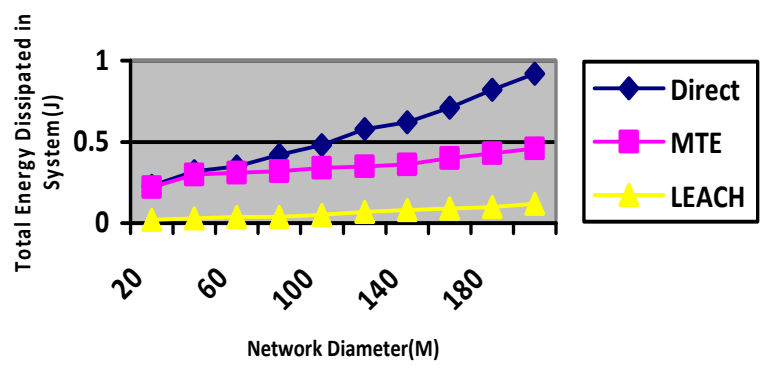

Figure 4. Total System Energy Dissipated

Figure 4 shows the total system energy dissipated by comparing LEACH [13] with two other protocols namely direct communication with the base station and minimum-energy multi-hop routing (MTE). Using a direct communication protocol by referring to Eq.3, each sensor sends its data directly to the 
base station. If the base station is far away from the nodes, this communication require high transmit power from each node as total energy consumption is proportional to $\mathrm{d}^{2}$, the transmission distance. In MTE, nodes route data destined for the base station through intermediate nodes (as referred to Eq.1). LEACH is a clustering based approach which shows better performance compared to two other approaches (Eq.2).

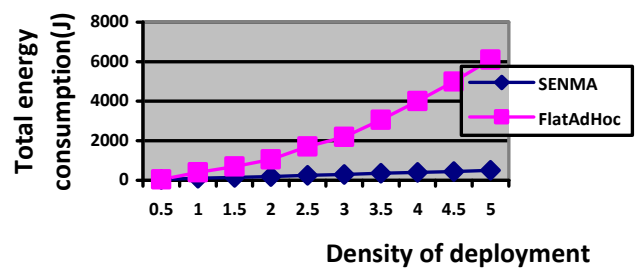

Figure 5. Total Energy Consumption

Figure 5 shows the total energy consumption over density of network for two different protocols, namely flatAdHoc network and SENMA [14]. SENMA deploys mobile agents(Eq.5\&6) for communication with the base station in which mobile agents are powerful hardware units which can be an aerial vehicle or ground vehicle. In this architecture, mobile agents are responsible to convey sensor data to the base station. Whereas in flatAdHoc architecture (Eq.1), sensor nodes are continuously consuming energy in order to route data from neighbor nodes. As seen in the figure 5, the deployment of mobile agent consumes lesser energy compared to flat architecture using multihop routing. This can be useful results to benchmark with the architecture proposed in this paper deploying mobile agent for routing as well as energy harvesting.

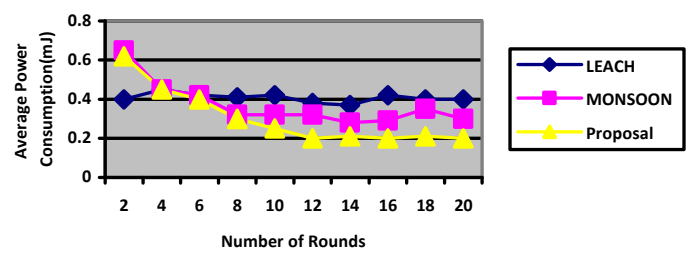

Figure 6. Average Power Consumption

Figure 6 shows average power consumption for three different protocols in which LEACH is a clustering based (Eq.3) approach, MONSOON [15] is a biologically inspired framework that consists of agents and middleware platform. The proposed framework (proposal)(Eq 5\&6) implements sensing data collection applications with each node deploying a Sensing Agent (SA) with a randomlygenerated behaviour policy. Based on the results presented with the mathematical analysis and comparison with other research works, the order of energy consumption from the highest to the lowest is: Direct $\rightarrow$ MTE/Flat (multihop) $\rightarrow \mathrm{LEACH}$ (cluster) -> SENMA/proposal (mobile) (figure 7).

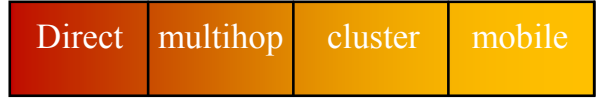

Figure 7. Flow of energy consumption

It is proven that mobile agent consumes the least energy for routing sensor data to the base station. And direct communication consumes the most energy for communicating sensor data to the base station. Direct transmission may not be a viable solution to reduce energy consumption in sensor networks. As seen in Eq.3, energy consumption will be affected by the transmission distance and energy consumption of nodes further away from the base station is four times more than nodes closer. These increases linearly as the radius increases and the energy consumption will be proportional to $d^{4}$ after the threshold value of transmission distance. Using a cluster head approach, sensors are only responsible for sensing the event and relaying data to the cluster head. Tremendous energy is saved as sensor nodes do not take part in routing and transmission of data as energy consumption is directly proportional to transmission distance. But as cluster head selection is on a rotational basis, sensor nodes would eventually deplete energy when it becomes the cluster head as it has to receive and send data from other nodes. Moreover based on Eq. 2 energy consumption using clustering approach is also affected by the width of the network since cluster head has to communicate using longer distance. Multihop communication ensures delivery of data as the data is forwarded by intermediate nodes. But multihop approach causes routing holes as nodes closer to the base station are heavily utilized and deplete energy quickly. The mobile agent based approach should be able to overcome the problem encountered by multihop as energy consumption in multihop is dependent $\left(\mathbf{2} \mathbf{L}^{2}\right)$ whereas in mobile approach it is dependent on $\left(\mathbf{L}^{2}\right)$. The discussion in the following section proves that energy consumption using mobile approach is two times lesser than multihop routing.

\section{Energy consumption and lifetime of mobile approach}

Theorem 1 : Total energy consumption using mobile agent is lower bounded by $1 / 2 e_{c}$, where $e_{c}$ is energy consumed by multihop routing

Proof: $_{\text {Assume }} \hat{C}=$ Energy $_{\text {cpu }} \quad \partial=$ Energy $_{\text {elec }} \quad \mu=$
Energy $_{\text {amp }}$ energy consumed by mobile $\mathrm{e}_{\mathrm{m}}=\frac{a L^{2}}{\pi r^{2}}\left(\mu X H^{2}+\hat{c}\right)$ energy using multihop $e_{C}=2 a L^{2}\left(\mu X d^{2}+\partial+{ }_{C}\right)$ 


\section{$\pi r^{2}$}

Looking at $e_{C}$ and $\mathrm{e}_{\mathrm{m}}, e_{C}>\mathrm{e}_{\mathrm{m}}$ or $\mathrm{e}_{\mathrm{m}}<1 / 2 e_{C}$ since $H<d$ and $e_{C}=1 / 2 \mathrm{e}_{\mathrm{m}}$ whereby $e_{C}$ is depending on distance $H$ and $\mathrm{e}_{\mathrm{m}}$ depending on distance $\mathrm{d}$,

Therefore,

$\boldsymbol{e}_{\boldsymbol{m}}<1 / 2 \mathbf{e}_{\mathrm{c}} \quad$ (energy mobile is lower bounded by $1 / 2$ energy multihop)

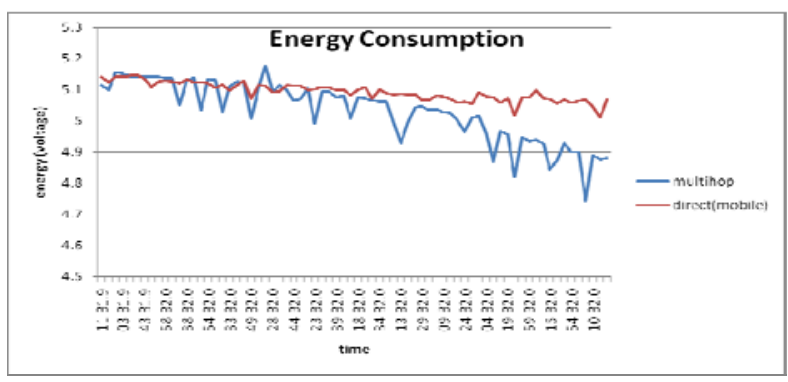

Figure 8: Energy consumption per node

Figure 8 outlines the results obtained from experiment using real sensors with each sensor boards attached to the standard battery. The energy consumption per node is traced by deploying the network in a multihop mechanism. The results obtained shows that mlutihop communication consumes more power and the energy in terms of voltage drops significantly as compared to direct communication. In this case the direct communication is configured as mobile architecture. Whereas figure 9 energy consumption for nodes placed near the mobile agent as well as nodes further away from the mobile agent. This shows that distance between mobile and sensor node is crucial for energy analysis.

Theorem 2: The lifetime of a node inversely proportional to distance $\mathrm{H}$ (between sensor node and mobile agent) and network width.

Proof

initial energy of a node $=\boldsymbol{E}$

energy per node using mobile agent $=e_{C}$

Therefore the lifetime of a node

And assuming

$$
=\boldsymbol{E} / \boldsymbol{e}_{\mathrm{C}}
$$

$$
\boldsymbol{e}_{C}=\frac{a L^{2}}{\boldsymbol{\pi} \boldsymbol{r}^{2}}\left(\mu \boldsymbol{X} \boldsymbol{H}^{2}+\partial\right)+{ }_{\hat{C}}
$$

$$
\begin{aligned}
\text { and lifetime per node } & =\boldsymbol{E} / \boldsymbol{a} \boldsymbol{L}^{2} \\
\boldsymbol{\pi} \boldsymbol{r}^{2} & \left(\mu \mathrm{X} \boldsymbol{H}^{2}+\partial+\right){ }_{\hat{C}} \\
& =\frac{\boldsymbol{\pi} \boldsymbol{r}^{2}(\boldsymbol{E})}{\boldsymbol{a} \boldsymbol{L}^{2}\left(\mu X \boldsymbol{H}^{2}+\partial+\right)_{\hat{C}}}
\end{aligned}
$$

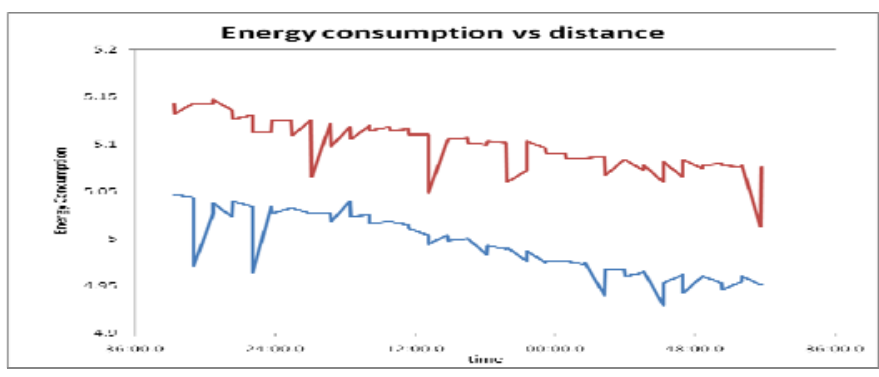

Figure 9. Energy consumption per node for different distance

\section{Case Study: Illegal Immigration Detection}

The mobile agent based approach discussed above will be applied into a prototype development into the illegal immigration detection system to prove with more applicable results. In line with the concern on protecting the country against illegal immigration and threat, a new mechanism using mobile wireless sensor technology is proposed. It is a self-healing wireless sensor network to detect any illegal immigrant moving into the country via forest or borders. This is specifically applicable immigrants entering the country through forest borders. I $t$ is predicted that most of the refugees and immigrants from Myanmar are entering the country through Malaysia-Thailand border. The sensor technology proposed is intelligent enough to detect any movements by using image processing techniques such as motion sensors, vision sensors and sound sensors to further analyze the profile.

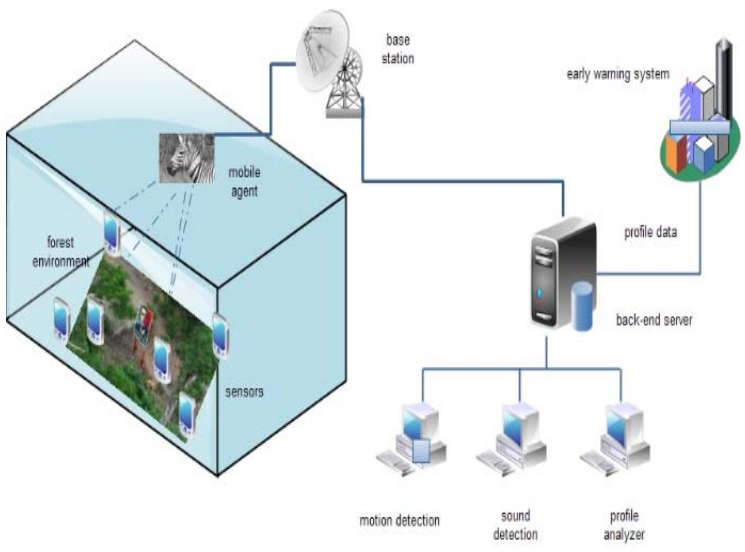

Figure 10. Illegal immigration system using mobile sensors

This is considered as micro-level technology that can detect any events in a smaller scale using image processing techniques as compared to remote sensing technology which is unable to detect events hidden from the satellite view. This technology works by static sensors being deployed to 
dynamically form a perimeter of network around the forest to detect any events as mentioned above. The sensed data is then forwarded to mobile agents (sensors) which are within the proximity of the area of the sensors. The mobile agent in this context works as a middleware to collect data from the static sensors and forward it to a base station for further action or early warning. Mobile agents can be aerial vehicles, ground vehicles with terminals and power generators that can hop around the network or it can be an airplane flying above the sensor field (airborne). Mobile agents do not need to be present all the time along with static sensors; they are only needed when it is necessary to collect data. Moreover mobile agents should have high data rate connection to base stations allowing faster dissemination of data.

Mobile agents are proposed in this context to shift away the processing and routing complexity from static sensors which are energy constrained. The use of optical and radar remote sensing (airborne sysnthetic aperture radar) is gaining popularity. Optical remote sensing has its disadvantage as it is restricted by clouds, haze and mist especially in high mountains. So a new technology using Mobile Wireless Sensor Technology can be a potential solution to combat the issues and problems discussed above. This research can even contribute to areas that need monitoring and early warning such as preserving natural resources, illegal logging, fire protection, soil erosion, tsunami warning, earthquake and others. Surveillance system could be another potential area where this research can be applied. In this case a security guard carrying a sensor embedded mobile device can be the agent collecting data from static sensors attached to buildings or forest for surveillance detection.

\section{Conclusion}

The proposed architecture in this paper using mobile agent as communication and energy harvesting agent should be a better solution for energy efficient operation in sensor networks. This can be further exploited by performing simulation of the protocol mentioned in section 4 and comparing with other protocols discussed in section 5 . In future mobile agent (sensors) should take the role of static sensors for forwarding sensor data to the base station as well as a source for energy harvesting and scavenging.

\section{References}

[1] T. Bokareva, N. Bulusu, S. Jha, "SASHA: Towards a self-healing hybrid sensor network architecture", in: Proceedings of The Second IEEE International Workshop on Embedded Networked Sensors, 2005.

[2] [4] M.S. Al-Fares, Z. Sun, H. Cruickshank, A Hierarchical Routing Protocol for Survivability in Wireless
Sensor Network (WSN). In Proceedings of the International MultiConference of Engineers and Computer Scientists, IMECS 2009, Vol 1.

[3] L. Tong, Q. Zhao, and S. Adireddy, "Sensor Networks with Mobile Agents," in Proc. 2003 Military Communications Intl Symp., (Boston, MA), Oct 2007.

[4] F. Dressler, B. Kuger, G. Fuchs, R. German, "SelfOrganization in Sensor Networks using Bio-Inspired Mechanisms," in $18^{\text {th }} \mathrm{ACM} / \mathrm{GI} / \mathrm{ITG}$ International Conference on Architecture of Computing SystemsSystem Aspects in Organic and Pervasive Computing (ARCS'05): Workshop Self-Organization and Emergence, Innsbruck, Austria, March 2005, pp 139-144.

[5] B. Haynes, M. Coles, and D. Azzi, "A Self-Healing Mobile Wireless Sensor Network using Predictive Reasoning”, Sensor Review, 28(4):326-333, 2008.

[6] Saad Ahmed Munir, Biao Ren, Weiwei Jiao, Bin Wang, Dongliang Xie, Jian Ma, "Mobile Wireless Sensor Network: Architecture and Enabling Technologies for Ubiquitous Computing", ainaw, vol. 2, pp.113-120, (AINAW'07), 2007

[7] Koutroullos M., Pitisllides A., "Biological and Nature Inspired Mechanisms for Adaptive and Robust SelfOrganization in Wireless Sensor Networks", EPL657, Projects 2007.

[8] Jan M. Rabaey,M. Josie Ammer, Julio L. da Silva Jr., Danny Patel, and Shad Roundy, "PicoRadio Supports Ad Hoc Ultra Low-Power Wireless Networking", IEEE Computer, vol.33, (no.7), IEEE Comput. Soc, July 2000. p. $42-8$.

[9] A. LaMarca, D. Koizumi, M. Lease, S. Sigurdsson, G. Borriello, W. Brunette, K. Sikorski, D. Fox, "PlantCare: An Investigation in Practical Ubiquitous Systems", Intel Research, IRS-TR-02-007, Jul. 23, 2002.

[10] Mohammed Rahimi, Hardik Shah, Gaurav S. Sukhatme,John Heidemann and D. Estrin. "Studying the Feasibility of Energy Harvesting in a Mobile Sensor Network", In IEEE Int'1

[11]F. Dressler and I. Carreras, "Advances in Biologically Inspired Information Systems - Models, Methods, and Tools", Studies in Computational Intelligence (SCI), vol. 69, Berlin, Heidelberg, New York, Springer, 2007

[12] Walker TS, Bais HP, Grotewold E, Vivanco JM (2003a) "Root exudation and rhizosphere biology", Plant Physiol 132: 44-51

[13]W.R.Heinzelman, A.Chandrakasan, and H.Balakrishnan, "Energy-Efficient Communication Protocol for Wireless Microsensor Networks", In Proceedings of the 33rd Hawaii International Conference on System Sciences, 2000

[14] L. Tong, Q. Zhao, and S. Adireddy, "Sensor Networks with Mobile Agents," in Proc. 2003 Military Communications Intl Symp., (Boston, MA), Oct 2007. 
[15]Pruet Boonma, and Junichi Suzuki, "MONSOON: A Coevolutionary Multiobjective Adaptation Framework for Dynamic Wireless Sensor Networks", In Proceedings of the 41st Hawaii International Conference on System Sciences, 2008.

[16]H. Sin, J. Lee, S. Lee, S. Yoo, S. Lee, J. Lee, Y. Lee, and S.Kim "Agent-based Framework for Energy Efficiency in Wireless Sensor Networks," Proceedings of World Academy of Science Engineering and Technology, vol.36, December 2008.

[17] Liu JS, Lin CH. Power efficiency clustering method with power limit constraint for sensor networks performance. In: Proceedings of the 2003 IEEE international performance, computing, and communications conference, vol. 9, Arizona, USA, 2003. p. 129-36.

[18] Priscilla Chen BO, Callaway E. Energy efficient system design with optimum transmission range for wireless ad-hoc networks. In: Proceedings of the 2002 IEEE international conference on communications, vol. 2, New York, USA, 2002. p. 945-52.

[19] J. Li, P. Mohapatra, Analytical modeling and mitigation techniques for the energy hole problems in sensor networks, Pervasive and Mobile Computing 3(8) (2007) 233-254. 